\title{
Is physician supervision of the capsaicin $8 \%$ patch administration procedure really necessary? An opinion from health care professionals
}

This article was published in the following Dove Press journal:

Journal of Pain Research

18 July 2013

Number of times this article has been viewed

\author{
Kai-Uwe Kern' \\ Janice England ${ }^{2}$ \\ Andrea Roth-Daniek ${ }^{3}$ \\ Till Wagner ${ }^{3}$ \\ 'Institute for Pain Medicine/Pain \\ Practice, Wiesbaden, Germany; \\ 2Pain Medicine and Anaesthesia, \\ The Christie National Health Service \\ Foundation Trust, Manchester, UK; \\ ${ }^{3}$ Pain Therapy and Palliative Care \\ Department, Medizinisches Zentrum \\ Städteregion Aachen, Aachen, \\ Germany
}

\begin{abstract}
Neuropathic pain is difficult to treat and can have a severe effect on quality of life. The capsaicin $8 \%$ patch is a novel treatment option that directly targets the source of peripheral neuropathic pain. It can provide pain relief for up to 12 weeks in patients with peripheral neuropathic pain. Treatment with the capsaicin $8 \%$ patch follows a clearly defined procedure, and patch application must be carried out by a physician or a health care professional under the supervision of a physician. Nonetheless, in our experience, nurses often take the lead role in capsaicin $8 \%$ patch application without the involvement of a physician. We believe that the nurse's key role is of benefit to the patients, as he or she may be better placed, because of time constraints and patient relationships, to support the patient through the application procedure than a physician. Moreover, a number of frequently prescribed drugs, including botulinum toxin and infliximab, can be administered by health care professionals without the requirement for physician supervision. Here we argue that current guidance should be amended to remove the requirement for physician supervision during application of the capsaicin $8 \%$ patch.
\end{abstract}

Keywords: capsaicin, neuropathic pain, topical, health care professional, physician, nurse

\section{Introduction to the capsaicin $8 \%$ patch}

Neuropathic pain is a difficult-to-treat, often chronic condition that can severely affect patients' quality of life. ${ }^{1,2}$ The capsaicin 8\% patch (Qutenza ${ }^{\mathrm{TM}}$; NeurogesX, Inc, San Mateo, CA, USA) is a novel treatment option that directly targets the source of peripheral neuropathic pain. The patch formulation contains a high concentration of capsaicin and has been approved for the treatment of peripheral neuropathic pain in nondiabetic adults. ${ }^{3}$ Capsaicin, the pungent ingredient in hot chili peppers, is a highly selective transient receptor potential vanilloid 1 agonist that produces reversible defunctionalization of nociceptive nerve fibers in the skin. ${ }^{4-8}$ This defunctionalization is, in part, a result of increases in intracellular calcium, neurochemical alterations at the peripheral ends of transient receptor potential vanilloid 1 expressing nerve fibers, and a reversible reduction in epidermal nerve fiber density. The overall effect is reduced spontaneous nerve activity and loss of responsiveness to many sensory stimuli. ${ }^{5,9}$ Capsaicin-induced defunctionalization is the mechanism behind the pain reducing effects of the capsaicin $8 \%$ patch, which have been demonstrated in clinical studies showing that a single application delivers prolonged pain relief for up to 12 weeks in patients with peripheral neuropathic pain. ${ }^{10-13}$
Correspondence: Kai-Uwe Kern Institute for Pain Medicine/Pain Practice Wiesbaden, Sonnenberger Strasse 68, 65193 Wiesbaden, Germany

Tel +4961120592636

Fax +49 6II 20592637

Email dr.kern@schmerzpraxis-wiesbaden.de 


\section{Application of the capsaicin $8 \%$ patch is well-defined}

The application of the capsaicin $8 \%$ patch is a clearly defined procedure. ${ }^{3,14}$ The patch is applied to the most painful areas of the skin for 30 minutes or 60 minutes, depending on the pain location. The area to be treated is indicated by the patient and defined by the physician before clearly marking it on the skin. To reduce any potential application-related discomfort, the treatment area may be pretreated with a topical anesthetic, or the patient might be administered an oral analgesic before patch application. ${ }^{3}$ The area to be treated is washed gently (removing topical anesthetic first, if used) and dried thoroughly before the patch or patches are applied. Each single-use patch is cut to match the size and shape of the treatment area, and the adhesive side of the patch is smoothed onto the skin as the release liner is slowly peeled away. ${ }^{14}$ It is essential to ensure the capsaicin $8 \%$ patch maintains close contact with the treatment area (eg, by using stretchable socks or bandages). After completion of the treatment time, the patch or patches are removed gently and slowly by rolling them inward to minimize the risk for aerosolization of capsaicin. Cleansing gel and gauze are used to remove any remaining capsaicin from the skin, and the area is gently washed with soap and water. ${ }^{14}$ Treatments with the capsaicin $8 \%$ patch may be repeated every 90 days, as warranted by the persistence or return of the pain.

A number of precautions should be taken when using capsaicin $8 \%$ patches: nitrile gloves should be worn at all times while handling the patch and cleaning treatment areas (latex gloves should not be worn as they do not provide adequate protection from the capsaicin); patches should not be placed or held near eyes or mucus membranes; and direct contact with patches, used gauze, or used cleansing gel should be avoided.

The patch application procedure is well tolerated by patients. ${ }^{3,10,13,15}$ Associated adverse events are mainly limited to transient localized reactions, with the most commonly reported adverse reactions being transient local applicationsite burning, pain, erythema, and pruritus. ${ }^{3,10,13,15}$ These application site reactions are mainly mild, nonserious, and resolve spontaneously within 7 days. ${ }^{16}$

\section{Current labeling and proposed change}

The capsaicin $8 \%$ patch is approved in Europe for the treatment of peripheral neuropathic pain in nondiabetic adults either as a monotherapy or in combination with other neuropathic pain medications. ${ }^{3}$ The current labeling states: "The QUTENZA ${ }^{\circledR}$ cutaneous patch should be applied by a physician or by a health care professional [HCP] under the supervision of a physician." 3

However, adherence to these guidelines prevents nurses or other trained HCPs from administering the patch without supervision. On the basis of the well-defined application procedure and favorable safety and tolerability profile of the capsaicin $8 \%$ patch, we believe this restriction is unnecessary. At our three centers alone, we have accrued experience with approximately 350 patch applications, and in our expert opinion, we believe the position surrounding the involvement of a physician should be clarified to remove the requirement for physician supervision.

\section{Current practice}

The capsaicin $8 \%$ patch was launched across Europe using a staggered approach to ensure its safe use after adequate training. After approval, an educational program with appropriate training materials for HCPs was initiated to minimize the potential for medication errors. This program was implemented successfully before launch in each country in which the capsaicin $8 \%$ patch has been made available.

As a group of physicians and nurses who are some of the most experienced users of the capsaicin $8 \%$ patch in Europe, we have found that nurses often take the lead role in carrying out patch application without the need for a physician present. Indeed, we feel the leading role of the nurse is beneficial to patients, as they are often more comfortable engaging in open dialogue with a nurse than with a physician.

Experience from German centers indicates that although the physician is involved in the diagnosis and assessment of neuropathic pain, the patch application procedure itself is mostly carried out by nurses. In the United Kingdom, the entire procedure may be carried out by either a physician or a nurse, but usually not both, and the treatment process is often undertaken by a nurse without input from a physician at any stage. In our experience, both nurses and physicians are equally capable of carrying out the procedure and providing support to help alleviate any patient fears or concerns about the treatment. It therefore seems unnecessary to potentially restrict clinic times or the number of patients treated on the basis of the availability of a physician to supervise. It is our opinion that it would be prudent for the efficient management of pain clinics for there to be the option for nurses to be able to carry out capsaicin $8 \%$ patch treatment without needing to consider having physician supervision. 


\section{Management of adverse effects}

The most common adverse effect we have observed while using the capsaicin $8 \%$ patch is application-related discomfort resulting from pain or a burning sensation that occurs during or after patch application. However, as experienced users of the capsaicin $8 \%$ patch, we have observed that tolerability of the patch in clinical practice is much better than was anticipated from the clinical trial experience. ${ }^{15,17-19}$ The requirement for medication to relieve application-associated discomfort was high in the clinical trial program, with 55\% of patients $(n=1696)$ receiving oral opioid analgesics on the day of treatment or up to 5 days posttreatment. ${ }^{15}$ However, in three separate retrospective analyses carried out in our patients $(n=165)$, we have observed that only $23 \%$ requested oral opioids during treatment, and only three $(1.8 \%)$ requested some analgesia (paracetamol, ibuprofen, or oral morphine) immediately after patch removal. ${ }^{17-19}$ We have also observed in these analyses that all patients except one, completed the intended duration of patch application; the one who asked for the patch to be removed 5 minutes early because of discomfort has since gone on to complete further patch applications with no incident. ${ }^{17-19}$

Although the majority of our patients do experience some application-related discomfort during or after patch application, we find this can be easily managed using distraction techniques. We also have found that providing reassurance and conversation to distract from possible discomfort caused by the patch can support the patient throughout the procedure and help alleviate any patient fears or concerns about the treatment. Local cooling (such as a cool compress) applied after patch removal is generally the most effective method of relieving the burning sensation that can be caused by treatment with the capsaicin $8 \%$ patch. ${ }^{14}$ In cases in which discomfort does require oral analgesia, in general, nonprescription medicines are used that do not specifically require dispensing by a physician or nurse prescriber. In addition, in our experience, the analgesia is prepared beforehand and is ready for the treating nurse or physician to provide as necessary. In rare cases in which prescription medication is required, the ability of a HCP to provide this without physician supervision would likely depend on country-, local- or even pain center-specific regulations.

In some patients, patch application is associated with a small transient increase in blood pressure (on average, a maximum increase of $<8.0 \mathrm{mmHg}$ ) or hypertension. In controlled clinical studies, there were four cases of hypertension $(<1 \%)$ and six cases of increased blood pressure $(<1 \%)$ deemed to be possibly related to capsaicin $8 \%$ patch treatment; as a consequence, they are classified as uncommon treatment-related adverse events. ${ }^{16}$ These adverse effects may be of concern in patients with uncontrolled hypertension or a recent history of cardiovascular events. ${ }^{3,20}$ However, provided the patient has well-controlled hypertension, this does not necessitate the presence of a physician. It is important that the physician take an accurate history at the time of prescribing the patch, which should be confirmed by the HCP before administration of the patch. Although blood pressure should be monitored during the treatment procedure, ${ }^{3}$ we have observed little change in the blood pressure of our patients during or after the patch application.

There is no evidence to suggest that increases in blood pressure or hypertension as a result of the patch are life threatening. In cases in which there is a high risk for increases in blood pressure or hypertension, we recommend a physician be available to consult and examine the patient if necessary. In cases such as these, the patient would be identified by physician examination before treatment. The physician would then be aware of the need to treat the patient or be available to assist the treating $\mathrm{HCP}$ if necessary.

\section{Other products with similar labeling}

In support of our opinion that the capsaicin $8 \%$ patch can be applied by trained HCPs, and that physician supervision is not always necessary, we refer to other drugs that are frequently administered by HCPs other than physicians.

In the United Kingdom, the summary of product characteristics (SmPC) for botulinum toxin has stated from launch that "The injections should be administered by appropriately trained personnel in hospital specialist centers." ${ }^{21}$ This is despite the risk, albeit minor, for potentially serious adverse events, including serious and/or immediate hypersensitivity reactions such as anaphylaxis and dyspnea; adverse effects related to the spread of toxin from the site of administration, which can result in death related to dysphagia, pneumonia, and/or significant debility; and the risk for procedure-related injuries, including infection, and bleeding. ${ }^{21}$ Another example is infliximab, the SmPC for which states that "Infliximab infusions should be administered by qualified [HCPs] trained to detect any infusion-related issues" since its launch. ${ }^{22}$ Nonetheless, drug infusion is an invasive procedure that can result in potentially serious infusion-related reactions. ${ }^{22}$ In addition, despite the risks of anaphylactic shock with vaccine administration (eg, Revaxis [Sanofi Pasteur MSD, Lyon, France] for tetanus, diphtheria, and polio; Menveo [Novartis Vaccines and Diagnostics, Cambridge, MA, USA] for meningitis; 
and Ambirix [GlaxoSmithKline UK, Uxbridge, UK] for hepatitis A), the SmPCs for these medications do not specify that a physician is required for administration..$^{23-25}$

To summarize, drugs with more invasive methods of administration and the potential for more serious adverse events than the capsaicin $8 \%$ patch are routinely administered by HCPs other than physicians, and without the requirement for administration under the supervision of a physician.

\section{Conclusion}

Several frequently prescribed drugs are currently licensed for administration by HCPs without the requirement for supervision by a physician, including botulinum toxin and infliximab. The application procedure of the capsaicin $8 \%$ patch is clearly defined, straightforward, and well tolerated, with few safety issues when carried out in the correct manner by a trained HCP. Further investigation into the requirement of physician supervision would be interesting, drawing on the experience of users of the capsaicin $8 \%$ patch in other centers and countries. Furthermore, it might be informative to perform a survey into how HCPs interpret the terminology of "physician supervision" and how this affects their routine practice with the capsaicin $8 \%$ patch. As experienced users of the capsaicin $8 \%$ patch, we believe that nurses play a key role in administration of the treatment. In addition, as well as being as capable as physicians, nurses are often responsible for carrying out the treatment procedure with no supervision or input. It is therefore our opinion that supervision by a physician is not necessary in most circumstances.

\section{Acknowledgments}

The authors thank Angela Sell from the Department of Pain Medicine, Portsmouth Hospitals National Health Service Trust, Portsmouth, United Kingdom, for her contribution to the content and review of the manuscript. Editorial assistance was provided by Adelphi Communications, supported by Astellas Europe Ltd.

\section{Disclosure}

Dr Kern is a consultant or presenter for Astellas, Berlin Chemie, Boehringer Ingelheim, Betapharm, Grünenthal, Lilly, Medi Bayreuth, and Mundipharma, and received LIIRA grant support for clinical research from Pfizer. Dr Wagner has received honoraria for educational lectures from Astellas Pharma Europe Ltd, Cephalon, Mundipharma, Grunenthal, Pfizer, TEVA-AWD, and MSD. Dr Kern and Dr Wagner have undertaken Qutenza ${ }^{\mathrm{TM}}$ speaker tours funded by Astellas Pharma Europe Ltd. Dr Wagner and Dr Roth-Daniek have received consultancy honoraria from Astellas Pharma Europe Ltd. Ms England has received consultancy honoraria from, has been a presenter for, and has undertaken Qutenza ${ }^{\mathrm{TM}}$ speaker tours funded by Astellas Pharma Europe Ltd. The authors declare no other conflicts of interest in this work.

\section{References}

1. Gilron I, Watson CP, Cahill CM, Moulin DE. Neuropathic pain: a practical guide for the clinician. CMAJ. 2006;175(3):265-275.

2. Schmader KE. Epidemiology and impact on quality of life of postherpetic neuralgia and painful diabetic neuropathy. Clin J Pain. 2002;18(6):350-354

3. Astellas Pharma Europe Ltd. Summary of Product Characteristics: Qutenza 179 Mg Cutaneous Patch. Chertsey, United Kingdom: Astellas Pharma Europe Ltd; 2013. Available from: http://ec.europa.eu/health/ documents/community-register/2013/20130311125355/anx_125355_ en.pdf. Accessed March 21, 2013.

4. Caterina MJ, Julius D. The vanilloid receptor: a molecular gateway to the pain pathway. Annu Rev Neurosci. 2001;24:487-517.

5. Anand P, Bley K. Topical capsaicin for pain management: therapeutic potential and mechanisms of action of the new high-concentration capsaicin 8\% patch. Br J Anaesth. 2011;107(4):490-502.

6. Nolano M, Simone DA, Wendelschafer-Crabb G, Johnson T, Hazen E, Kennedy WR. Topical capsaicin in humans: parallel loss of epidermal nerve fibers and pain sensation. Pain. 1999;81(1-2):135-145.

7. Premkumar LS, Sikand P. TRPV1: a target for next generation analgesics. Curr Neuropharmacol. 2008;6(2):151-163.

8. Bley KR. TRPV1 agonist approaches for pain management. In: Gomtsyan A, Faltynek CR, editors. Vanilloid Receptor TRPV1 in Drug Discovery. Hoboken NJ: John Wiley \& Sons, Inc; 2010:325-347.

9. Wallace M, Pappagallo M. Qutenza ${ }^{\circledR}$ : a capsaicin $8 \%$ patch for the management of postherpetic neuralgia. Expert Rev Neurother. 2011;11(1):15-27.

10. Backonja M, Wallace MS, Blonsky ER, et al; NGX-4010 C116 Study Group. NGX-4010, a high-concentration capsaicin patch, for the treatment of postherpetic neuralgia: a randomised, double-blind study. Lancet Neurol. 2008;7(12):1106-1112.

11. Backonja MM, Malan TP, Vanhove GF, Tobias JK; C102/106 Study Group. NGX-4010, a high-concentration capsaicin patch, for the treatment of postherpetic neuralgia: a randomized, double-blind, controlled study with an open-label extension. Pain Med. 2010;11(4): 600-608.

12. Irving GA, Backonja MM, Dunteman E, et al; NGX-4010 C117 Study Group. A multicenter, randomized, double-blind, controlled study of NGX-4010, a high-concentration capsaicin patch, for the treatment of postherpetic neuralgia. Pain Med. 2011;12(1):99-109.

13. Simpson DM, Brown S, Tobias J; NGX-4010 C107 Study Group. Controlled trial of high-concentration capsaicin patch for treatment of painful HIV neuropathy. Neurology. 2008;70(24):2305-2313.

14. England J, Wagner T, Kern KU, Roth-Daniek A, Sell A. The capsaicin $8 \%$ patch for peripheral neuropathic pain. Br J Nurs. 2011;20(15): 926-931.

15. Peppin JF, Majors K, Webster LR, Simpson DM, Tobias JK, Vanhove GF. Tolerability of NGX-4010, a capsaicin $8 \%$ patch for peripheral neuropathic pain. J Pain Res. 201;4:385-392.

16. European Medicines Agency. CHMP Assessment Report for QUTENZA (EPAR). London: European Medicines Agency; 2009. Available from: http://www.ema.europa.eu/docs/en_GB/document_library/ EPAR_-_Public_assessment_report/human/000909/WC500040450. pdf. Accessed November 5, 2012.

17. Bhaskar A, England J, Lowe J. Management of neuropathic pain using the capsaicin $8 \%$ patch in patients with cancer. Proceedings of the 7 th World Research Congress of the European Association of Palliative Care; June 7-9, 2012; Trondheim, Norway. 
18. Kern K-U, Poole C, Nowack W. Treatment of neuropathic pain with the capsaicin $8 \%$ patch (QUTENZA ${ }^{\mathrm{TM}}$ ): Is pre-treatment with lidocaine necessary? Proceedings of Pain in Europe VII, 7th Congress of the European Federation of IASP Chapters; September 21-24, 2011; Hamburg, Germany.

19. Wagner T, Poole C, Roth-Daniek A. The capsaicin $8 \%$ patch for neuropathic pain in clinical practice: A retrospective analysis. Proceedings of the 15 th Congress of the European Federation of Neurological Societies; September 10-13, 2011; Budapest, Hungary.

20. Vanhove GF, Wallace M, Irving G, Backonja M, Webster LR, Tobias JK. Integrated safety analyses of NGX-4010, an $8 \%$ capsaicin patch, in patients with peripheral neuropathic pain. Proceedings of the 13 th World Congress on Pain; August 29-September 2, 2010; Montreal, Canada.

21. Summary of product characteristics: BOTOX 50 units. [Webpage on the Internet]. Allergan Ltd; 2010. Available from: http://www.medicines. org.uk/EMC/medicine/20564/SPC/BOTOX+50+Units/. Accessed November 5, 2012.
22. Summary of product characteristics: Remicade $100 \mathrm{mg}$ powder for concentrate for solution for infusion. [Webpage on the Internet]. Schering-Plough Ltd; 2011. Available from: http://www.medicines. org.uk/EMC/medicine/3236/SPC/Remicade+100mg+powder+for+ concentrate+for+solution+for+infusion/. Accessed November 5, 2012.

23. Summary of product characteristics: Ambirix suspension for injection. [Webpage on the Internet]. GlaxoSmithKline; 2011. Available from: http://www.medicines.org.uk/emc/medicine/20491/SPC/Ambirix+sus pension+for+injection/. Accessed November 5, 2012.

24. Summary of product characteristics: Menveo Group A, C, W135 and Y conjugate vaccine. [Webpage on the Internet]. Novartic Vaccines; 2011. Available from: http://www.medicines.org.uk/EMC/medicine/22866/ $\mathrm{SPC} /$ Menveo+Group $+\mathrm{A} \% 2 \mathrm{c}+\mathrm{C} \% 2 \mathrm{c}+\mathrm{W} 135+\mathrm{and}+\mathrm{Y}+$ conjugate+vacci ne/. Accessed November 5, 2012.

25. Summary of product characteristics: REVAXIS. [Webpage on the Internet]. Sanofi Pasteur MSD Limited; 2008. Available from: http://www. medicines.org.uk/emc/document.aspx?documentId=15259. Accessed November 5, 2012.
Journal of Pain Research

\section{Publish your work in this journal}

The Journal of Pain Research is an international, peer-reviewed, open access, online journal that welcomes laboratory and clinical findings in the fields of pain research and the prevention and management of pain. Original research, reviews, symposium reports, hypothesis formation and commentaries are all considered for publication.

\section{Dovepress}

The manuscript management system is completely online and includes a very quick and fair peer-review system, which is all easy to use. Visit http://www.dovepress.com/testimonials.php to read real quotes from published authors. 